\title{
Speckle Noise Reduction of Medical Ultrasound Images using Bayesshrink Wavelet Threshold
}

\author{
K. Karthikeyan \\ Assistant Professor, Department of Information Technology, \\ Dr. SNS Rajalakshmi College of Arts \& Science, \\ Coimbatore - 49, TN, India.
}

\author{
Dr. C. Chandrasekar \\ Associate Professor, Department of \\ Computer Science, Periyar University, \\ Salem - 11, TN, India.
}

\begin{abstract}
In diagnosis of diseases Ultrasonic devices are frequently used by healthcare professionals. The main problem during diagnosis is the distortion of visual signals obtained which is due to the consequence of the coherent of nature of the wave transmitted. These distortions are termed as 'Speckle Noise'. The present study focuses on proposing a technique to reduce speckle noise from ultrasonic devices. This technique uses a hybrid model that combines fourth order PDE based anisotropic diffusion, linked with SRAD filter and wavelet based BayesShrink technique. The proposed filter is compared with traditional filters and existing filters using anisotropic diffusion. Experimental results prove that the proposed method is efficient in reaching convergence quickly and producing quality denoised images.
\end{abstract}

Keywords: Anisotropic Diffusion, BayesShrink, Fourth Order PDE, Speckle denoising, SRAD Filter, Wavelet Based.

\section{INTRODUCTION}

The medical imaging devices namely X-ray, CT/MRI and ultrasound are producing abundant images which are used by medical practitioners in the process of diagnosis. The main problem faced by them is the noise introduced due to the consequence of the coherent nature of the wave transmitted. These noises corrupt the image and often lead to incorrect diagnosis. Each of these medical imaging devices is affected by different types of noise. For example, the x-ray images are often corrupted by Poisson noise, while the ultrasound images are affected by Speckle noise. Speckle is a complex phenomenon, which degrades image quality with a backscattered wave appearance which originates from many microscopic diffused reflections that passing through internal organs and makes it more difficult for the observer to discriminate fine detail of the images in diagnostic examinations. Thus, denoising or reducing these speckle noise from a noisy image has become the predominant step in medical image processing.

Multi-look process and spatial filtering are the two techniques of reducing speckle noise. Multi-look process is used at the data acquisition stage while spatial filtering is used after the data is stored. Among the two any method can be used to remove the speckle noise, but they should preserve radiometric information, edge information and spatial resolution ([1], [21]). These conditions are met by speckle noise reduction technique.

Many methods have been developed to reach the above mentioned objectives, ([20], [33], [29], [14]). The speckle noise reduction techniques use traditional filters like lee, kaun, frost, median, and hybrid filters and wavelet filters.
Performance comparison of all these wavelets has also been investigated ([26], [35], [23]). In the past few decades the use of wavelet based denoising techniques have gained more attention by researchers ([15], [7], [10], [3]). Figure 1 depicts the general process of a Wavelet based Speckle Denoising (WSD) model.

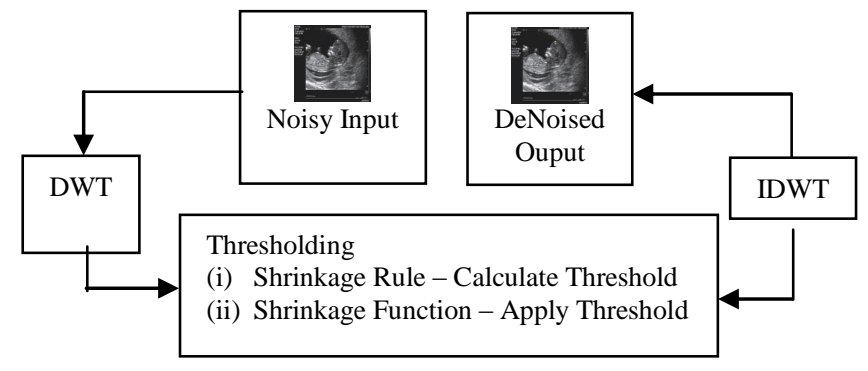

Figure 1 : WSD Model

The first step in the proposed method is a linear forward Discrete Wavelet Transform (DWT), followed by a non-linear thresholding step and the final step performs a linear Inverse Discrete Wavelet Transform (IDWT). The thresholding step comprises of two subtasks, (i) threshold technique selection and (ii) applying using a threshold operator. Two threshold operators used during denoising are soft thresholding and hard thresholding. Soft thresholding has major advantages over hard thresholding. Soft thresholding reduces the abrupt sharp changes and provides an image whose quality is not affected. Due to these advantages, soft thresholding is more frequently used. Once the thresholding operator has been defined, the next step is to address the problem of selecting the corresponding threshold.

The selection of threshold is the most important step in any WSD model. Careful selection is needed because a small threshold will produce an image which is still noisy, while a large threshold destroys details and produces blurs and artifacts. Two types of thresholding techniques namely, Universal Thresholding (UT) and Subband Adaptive Thresholding (SA) exists. Based on this, three shrinkage techniques used are Visushrink [9], SureShrink [8] and BayesShrink [5]. VisuShrink uses a universal threshold ( $t=\sigma \sqrt{2 \log \mathrm{n}}$ ), while SureShrink uses a combination of Universal Threshold and SURE Threshold, which is derived from Stein's Unbiased Risk Estimator. BayesShrink performs soft thresholding, with the data-driven, subband dependent threshold. The threshold is driven in a Bayesian framework, with a Generalized Gaussian Distribution (GGD) for the wavelet coefficients in each detail subband. Out of the three, 
BayesShrink is effective in denoising problem domain than VisuShrink and SureShrink ([22], [4]).

Anisotropic diffusion filter is another denoising technique which is equally gaining popularity [41]. Anisotropic diffusion was initially introduced by [27] and has been improved by several manners. Problems faced by the initial Anisotropic diffusion filter and its variants are;

(i) they cause blocky effects in images

(ii) they destroy structural and spatial neighbourhood information [28] and

(iii) they are slow in reaching a convergence stage.

Hybrid varieties were developed to solve these disadvantages ([18], [31], [32]). Eventhough, these hybrid models produce excellent results when compared with stand-alone anisotropic diffusion, they come with the defect of removing finer details of an image like edges, sharp corners and thin lines [13]. A hybrid denoising technique to remove noise from molecular images was developed [32]. This work improved the filter developed by [18], which used anisotropic diffusion and Median filter to reduce noise. The Rajan Hybrid Model (RHM) improved this method by using a combination of $4^{\text {th }}$ order PDE (Partial Differential Equation) anisotropic diffusion and a relaxed median filter [38] to remove the noise. The RHM was successful in removing the noise and had less blocking effects. The drawback still faced is the slow convergence to remove the noise. The reason behind this is that the convergence time for denoising is directionally proportional to the image noise level. In the case of anisotropic diffusion, as iteration continues, the noise level in image decreases (till it reaches the convergence point), but in a slow manner. But in the case of Bayesian shrinkage, it just cut the frequencies above the threshold in a single step. Decrease in convergence time has a direct impact on image quality. This model of using BayesShrink with Anisotropic filter was proposed by [30] and they named their denoising model as WEAD. Even though this work reduced the blocking artifacts and fast convergence, improvements are still needed. This paper proposes a novel method that improves the RHM and WEAD model, by introducing a new step. From the review, it is understood that Frost and Lee filter can be best combined with anisotropic diffusion for denoising. This method is used by the famous traditional despeckling algorithm proposed by [41]. In this study, the PDE used by Speckle Reducing Anisotropic Diffusion [SRAD] is modified to use a $4^{\text {th }}$ order PDE based Anisotropic diffusion, followed by Frost Filter which is then fed to BayesShrink technique to obtain a denoised image.

This paper is organized in four sessions which comprises of the introduction of the topic, introduction of the BayesShrink soft thresholding, Kaun Filter and Anisotropic diffusion, processes of the proposed model, the results of the proposed denoising model which was compared with BayesShrink, Anisotropic filter, SRAD filter, RHM and WEAD and finally the conclusion of the work.

\section{FILTERS USED IN THE PROPOSED MODEL}

In the present work, three techniques, namely, SRAD filter, anisotropic diffusion and BayesShrink soft thresholding are combined to form a hybrid speckle denoising model. These techniques are explained below.

\subsection{BayesShrink}

The goal of BayesShrink method is to minimize the Bayesian risk, and hence its name, BayesShrink. It uses soft thresholding and is subband-dependent, which means that thresholding is done at each band of resolution in the wavelet decomposition. Like the SureShrink procedure, it is smoothness adaptive. The Bayes threshold, $\mathrm{t}_{\mathrm{B}}$, is defined as

$$
\mathrm{t}=\sigma^{2} / \sigma_{\mathrm{s}}^{2}
$$

where $\sigma^{2}$ is the noise variance and $\sigma_{s}^{2}$ is the signal variance without noise. The noise variance $\sigma^{2}$ is estimated from the subband HH1 by the median estimator shown in Equation (2). From the definition of additive noise,

$$
\mathrm{w}(\mathrm{x}, \mathrm{y})=\mathrm{s}(\mathrm{x}, \mathrm{y})+\mathrm{n}(\mathrm{x}, \mathrm{y})
$$

Since the noise and the signal are independent of each other, it can be stated that

$$
\sigma^{2}{ }_{\mathrm{w}}=\sigma_{\mathrm{s}}^{2}+\sigma_{\mathrm{w}}^{2}
$$

$\sigma^{2} w$ can be computed using Equation (4). From this the variance of the signal, $\sigma^{2}$ s can be computed using Equation (5).

$$
\begin{aligned}
& \sigma_{\mathrm{w}}^{2}=\frac{1}{\mathrm{n}^{2}} \sum_{\mathrm{x}, \mathrm{y}=1}^{\mathrm{n}} \mathrm{w}^{2}(\mathrm{x}, \mathrm{y}) \\
& \sigma_{\mathrm{s}}=\sqrt{\max \left(\sigma_{\mathrm{w}}^{2}-\sigma^{2}, 0\right)}
\end{aligned}
$$

with $\sigma^{2}$ and $\sigma_{s}^{2}$, the Bayes threshold is computed from Equation (1).

\subsection{Speckle Reducing Anisotropic Diffusion (SRAD)}

Anisotropic Diffusion is a nonlinear smoothing filter [12] which uses a variable conductance term, that controls the contrast of the edges that influence the diffusion. This filter has the ability to preserve edges, while smoothing the rest of the image to reduce noise [34]. The anisotropic diffusion has been used by several researchers in image restoration [24] and image recovery [36]. SRAD [41] is an edge-sensitive Partial Differential Equation (PDE) anisotropic diffusion approach to reduce speckle noise in images. The anisotropic filtering in SRAD simplifies image features to improve image segmentation and smoothes the image in homogeneous area while preserving edges and enhances them. It reduces blocking artifacts by deleting small edges amplified by homomorphic filtering. SRAD equation for an image $u$ is given by the Equation (6).

$\operatorname{SRAD}\left(u^{\prime}\right)=u t+1=u t+\frac{\Delta t}{4} \operatorname{div}\left(g\left(\operatorname{ICOV}\left(u^{\prime}\right)\right) \times \nabla u^{\prime}\right)$

where $\mathrm{t}$ is the diffusion time index, $\Delta \mathrm{t}$ is the time step responsible for the convergence rate of the diffusion process (normally in the range 0.05 to 0.25$), \mathrm{g}($.$) is the diffusion$ function and is given by equations (7) and (8).

$$
\mathrm{G}\left(\operatorname{ICOV}\left(\mathrm{u}^{\prime}\right)\right)=\mathrm{e}-(\mathrm{P})
$$




$$
P=\frac{\left(\frac{\operatorname{ICOV}\left(\mathrm{u}^{\prime}\right)}{\mathrm{q}^{\mathrm{t}}}\right)^{2}-1}{1+\left(\mathrm{q}^{\mathrm{t}}\right)^{2}}
$$

where $\mathrm{q}^{\mathrm{t}}$ is the measure of speckle coefficient of variation in a homogenous region of the image.

The performance of SRAD is superior to the traditional anisotropic diffusion filters. However, SRAD has the disadvantage that the diffusion time increases with the image features and it is already known that when diffusion time increases the image quality of the denoised image decreases.

\subsection{Fourth Order PDEs and Anisotropic Diffusion}

Recently, non-linear fourth order PDEs are used effectively in the field of noise reduction ([11], [40], [19], [38]), because they are faster in denoising and create a richer set of functional behaviour that can be exploited during image enhancement. The $\mathrm{L}^{2}$-curvature gradient flow method of [40] is used and given in Equation (9).

$$
\frac{\partial \mathrm{u}}{\partial \mathrm{t}}=-\nabla^{2}\left[\mathrm{c}\left(\left|\nabla_{\mathrm{u}}^{2}\right|\right) \nabla_{\mathrm{u}}^{2}\right]
$$

here $\nabla^{2} u$ is the Laplacian of the image u. Hence, the Laplacian of an image at a pixel is zero if the image is planar in its neighborhood, the PDE attempt to remove noise and preserve edges by approximating an observed image with a piecewise planar image. The desirable diffusion coefficient c(.) should be such that Equation (9) diffuses more in smooth areas and less around less intensity transitions, so that small variations in image intensity such as noise and unwanted texture are smoothed and edges are preserved. Another objective for the selection of $\mathrm{c}($.$) is to incur backward$ diffusion around intensity transitions so that edges are sharpened, and to assure forward diffusion in smooth areas for noise removal ([39], [32]).

Several diffusivity functions can be used in Wavelet Shrinkage and Nonlinear Diffusion [25]. Some of them are Linear diffusivity [17], Charbonnier diffusivity [6], Weickert diffusivity, TV diffusivity [2], BFB diffusivity [16] and Perona-Malik diffusivity [27]. The present study uses Perona-Malik diffusivity as given in Equation (10).

$$
\mathrm{c}(\mathrm{s})=\frac{1}{1+\left(\frac{\mathrm{s}}{\mathrm{k}}\right)^{2}}=\exp \left[-\left(\frac{\mathrm{s}}{\mathrm{k}}\right)^{2}\right]_{\mathrm{s}=0}
$$

The Equation (9) was associated with the following energy functional

$$
E(u)=\int_{\Omega} f\left(\left|\nabla^{2} u\right|\right) d x d y
$$

where $\Omega$ is the image support and $\nabla^{2}$ denotes Laplacian operator. Since $\mathrm{f}\left(\left|\nabla^{2} \mathrm{u}\right|\right)$ is an increasing function of $\left|\nabla^{2} \mathrm{u}\right|$, its global minimum is at $\left|\nabla_{\mathrm{u}}^{2}\right|=0$. Consequently, the global minimum of $E(u)$ occurs when

$$
\left|\nabla^{2} u\right| \equiv 0 \quad \text { for } \text { all }(x, y) \in \Omega
$$

A planar image obviously satisfies [30], hence is a global minimum of $E(u)$. Planar images are the only global minimum of $E(u)$ if

$$
\mathrm{f}^{\prime \prime}(\mathrm{S}) \geq \text { for all } \mathrm{s} \geq 0
$$

because the cost functional $E(u)$ is convex under this condition [40]. Therefore, the evolution of (9) is a process in which the image is smoothed more and more until it becomes a planar image. But in the case of second order anisotropic diffusion $f^{\prime \prime}(\mathrm{s})$ may not be greater than zero for all $s$ and as a result the image is evolved towards a step image and that is why it suffers from blocky effects.

\section{PROPOSED DESPECKLING MODEL}

The existing models suffer from blocky effects, which in the present study are removed by using fourth order PDE. This technique preserves edges and boundaries which are more stable through the scale ' $t$ '. Another difficulty faced by the existing models is that, if the image is very noisy, the gradient $\nabla \mathrm{u}$ will be very large, and as a result, the function $\mathrm{c}($.$) will be$ close to zero at almost every point. When the smoothing is introduced the noise will remain consequently. This difficulty is solved by using a suitable filter that can reduce noise and at the same time be combined with fourth order PDE based anisotropic diffusion. So the SRAD filter was considered and to speed up the convergence, BayesShrink is used.

The main objective is to reduce the blocking artifacts produced by reducing the number of iterations required to reach a convergence point. The iteration process in the proposed model will continue till the input signal ' $y$ ' is converged to the output signal ' $Y$ '. The convergence process of an image, while using $\mathrm{P}-\mathrm{M}$ anisotropic diffusion is shown in Figure 2 [30].

The noisy image is enhanced at $\mathrm{P}$ which is the convergence point (Figure 2a). The main aim of the proposed work is to pull $\mathrm{P}$ towards top-left of the chart, thus achieving a better PSNR in minimum number of iterations (Figure $2 b$ ). For this purpose BayesShrinkage is used. The method of WEAD is enhanced in two ways. The first is a fourth order PDE, which is used instead of second order PDE and the SRAD filter is used to reduce the blocking artifacts and improve the quality of the despeckled image.

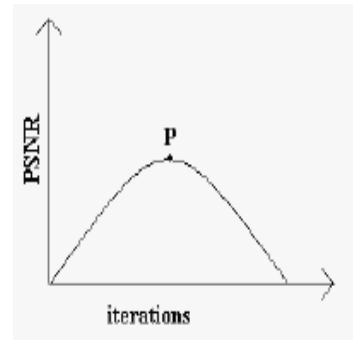

2a

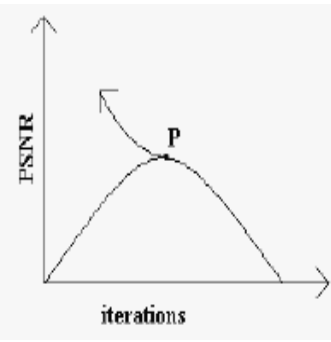

$2 b$
Figure 2 : Convergence process of P-M Anisotropic Diffusion 
The proposed model is a fourth order PDE, Anisotropic diffusion with SRAD filter combined with BayesShrink wavelet denoising model (WASD). The WASD model is a three step process as given in Figure 3 .

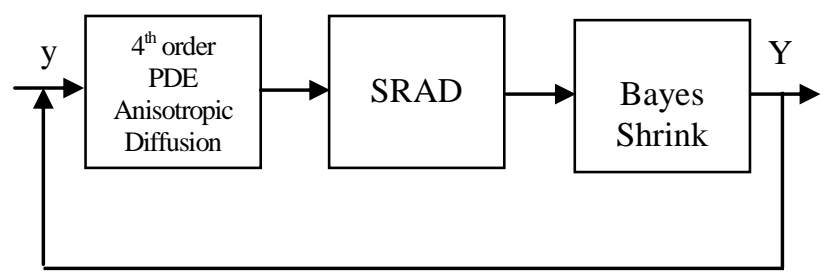

Figure 3 : Proposed WASD

\section{EXPERIMENTAL RESULTS}

Several experiments were conducted to evaluate the proposed despeckling model. The performance metrics used are (i) Peak Signal to Noise Ratio (PSNR) and (ii) Denoising Time. PSNR is a quality measurement between the original and a denoised image. The higher the PSNR, the better is the quality of the compressed or reconstructed image.

To compute PSNR, the block first calculates the MeanSquared Error (MSE) and then the PSNR (Equation 14).

$\mathrm{PSNR}=10 \log _{10}\left[\frac{\mathrm{R}^{2}}{\mathrm{MSE}}\right]$

where MSE $=\frac{\sum_{M, N}\left[I_{1}(m, n)-I_{2}(m, n)\right]^{2}}{M * N}$ where $M$ and

$\mathrm{N}, \mathrm{m}$ and $\mathrm{n}$ are number of rows and columns in the input and output image respectively

Denoising time denotes the time taken for the algorithm to perform the despeckling procedure. Further, the results of the proposed model was compared with the four traditional filters namely, Median, Lee, Frost, Kaun and SRAD and also with RHM and WEAD. The despeckling models were tested with the test image (grayscale) of $256 \times 256$ size (Figure 4). The proposed models were implemented using MATLAB 7.3 and were tested on Pentium IV machine with 512 MB RAM.
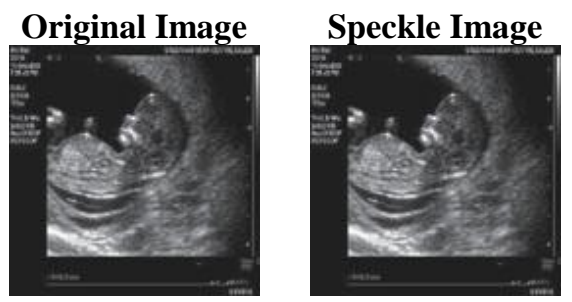

Figure 4 : Test Image

The Peak Signal to Noise Ratio obtained for the different filters are tabulated (Table 1). The efficiency percentage is calculated as the efficiency obtained while comparing the PSNR between the original, noisy image and original, despeckled image. The PSNR value of original and Speckle noised image is $22.95 \mathrm{~dB}$. The efficiency is calculated using Equation 15.

$$
\begin{gathered}
\text { Efficiency }(\%)= \\
\frac{((\mathrm{PSNR}(\mathrm{OI}, \mathrm{SNI})-\mathrm{PSNR}(\mathrm{OI}, \mathrm{DI})}{(\mathrm{PSNR}(\mathrm{OI}, \mathrm{SNI})} \times 100
\end{gathered}
$$

where $\mathrm{OI}$ is the original image, $\mathrm{SNI}$ is the speckle noise image and DI is the denoised image.

Table 1

PSNR Performance

\begin{tabular}{|l|c|c|}
\hline \multicolumn{1}{|c|}{ Filter } & PSNR & Efficiency (\%) \\
\hline Lee & 25.74 & 10.84 \\
\hline Frost & 25.61 & 10.39 \\
\hline Median & 25.15 & 8.75 \\
\hline Kaun & 23.68 & 3.08 \\
\hline Anisotropic Diffusion & 24.46 & 6.17 \\
\hline SRAD & 26.77 & 14.27 \\
\hline RHM & 29.34 & 21.78 \\
\hline Bayes & 52.91 & 56.62 \\
\hline WEAD & 52.90 & 56.62 \\
\hline Proposed & 70.96 & 67.66 \\
\hline
\end{tabular}

From the Table it is evident that the proposed method is efficient in image quality when compared to all the traditional filters. The high PSNR obtained by the proposed model indicates that it is the best choice for removing speckle noise from ultrasound images. Figure 5 visually compares the result between the different shrinkage methods.

The time taken to despeckle an noisy image is shown in Figure 6. The figure again proves that the proposed model is quick in removing speckle noise. From the results projected, it is clear that the proposed system outperforms all the traditional models in terms of quality and time.

\section{CONCLUSION}

Image denoising has become a crucial step for correct diagnosis. The current need of healthcare industries is to preserve useful diagnostic information with minimum noise. Ultrasound images often suffer with a special type of noise called speckle. Introduction of speckle degrades the image contrast and block out the underlying anatomy. In order for the medical practitioners to achieve correct diagnosis, the ultrasound images have to be despeckled. This study proposes a new hybrid model which is a combination of anisotropic diffusion combined with SRAD filter and BayesShrink thresholding. The experimental results prove that the proposed model produce images which are cleaner and smoother and at the same time kept significant details, resulting in a clearer an appealing vision. Moreover, the proposed method is fast at reaching the convergence, which has a direct impact on noise reduction. 

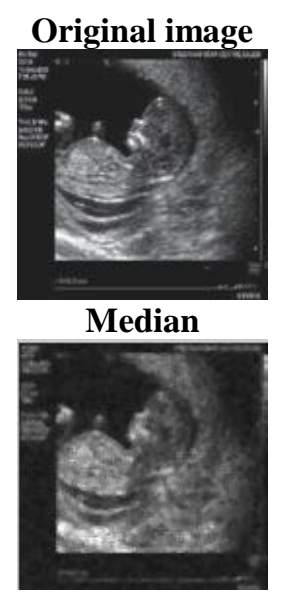

RHM
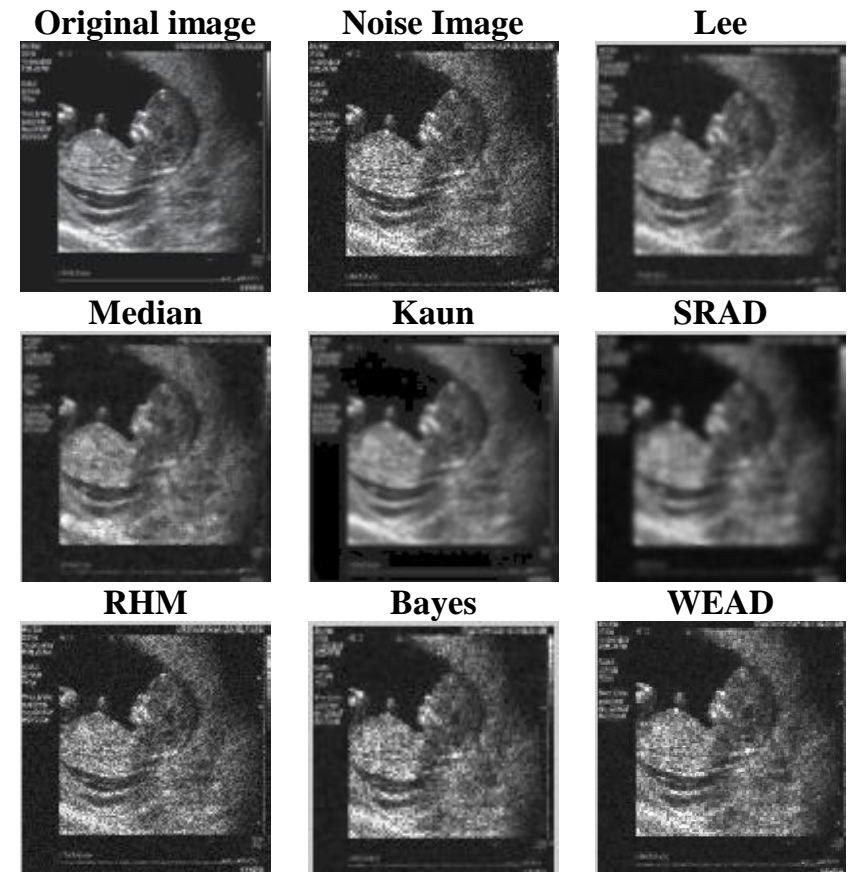

Figure 5 : Visual Results

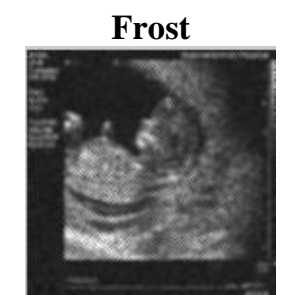

Anisotropic Diffusion

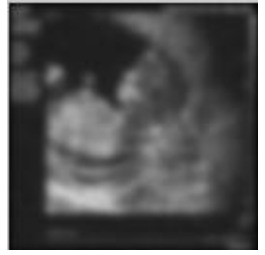

Proposed

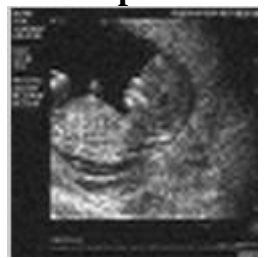

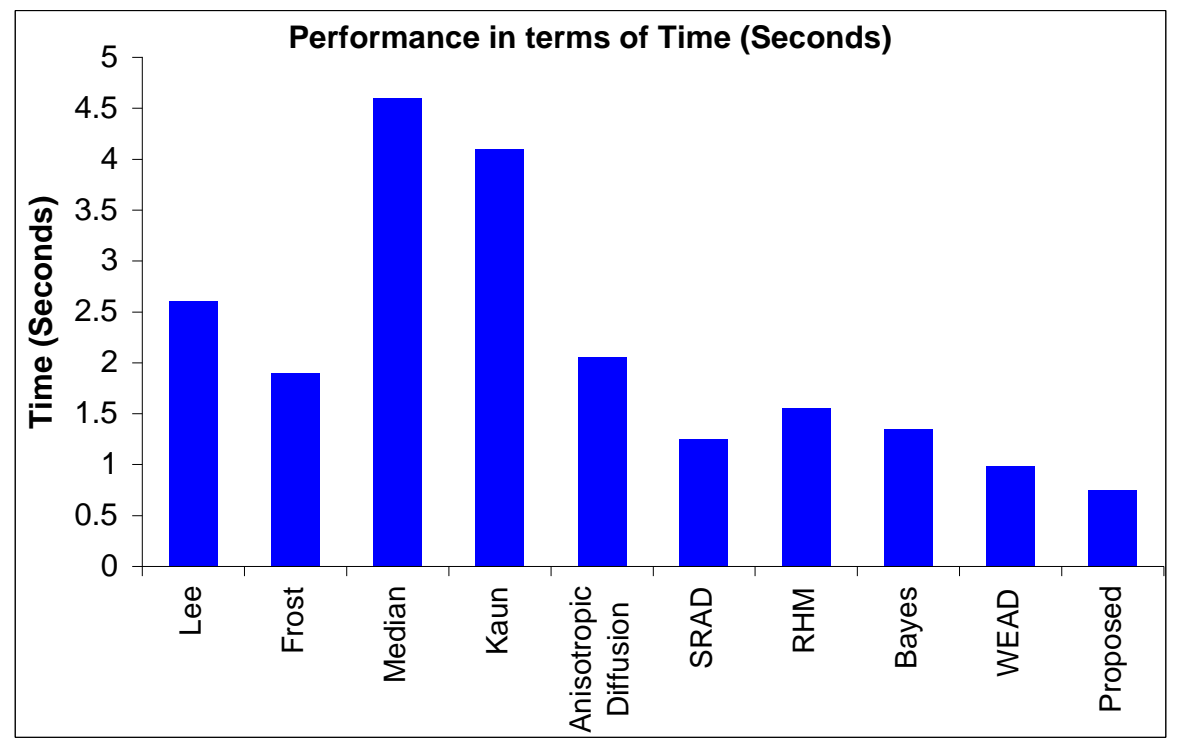

Figure 6 : Time Per Performance

\section{REFERENCES}

[1] Abdullah, H.N., Hasan, M.F. and Tawfeeq, Q.S. (2008), Speckle noise reduction in SAR images using Double-Density Dual Tree DWT, Asian Journal of Information Technology, Vol. 7, No. 7, pp. 281-284.

[2] Andreu, F., Ballester, C., Caselles, V. and Mazn, J.M. (2001), Minimizing total variation flow,
Differential and Integral Equations, Vol. 14, pp 321-360.

[3] Antoniadis, A. and Bigot, J. (2001), Wavelet Estimators in Nonparametric Regression: A Comparative Simulation Study, Journal of Statistical Software, Vol 6, No. I, P. 06.

[4] Arivazhagan, S., Deivalakshmi, S. and Kannan, K. (2007), Performance Analysis of Image Denoising System for different levels of Wavelet 
decomposition, International Journal of Imaging Science and Engineering (IJISE), Vol.1,No.3, pp. 104-107.

[5] Chang, G., Yu, B. and Vetterli, M. (2000),Adaptive Wavelet Thresholding for Image Denoising and Compression, IEEE Trans of Image Processing, Vol. 9, No. 9, pp. 1532-1546.

[6] Charbonnier, P., Aubert, G., Feraud, L.B. and Barlaud, M. (1994), Two deterministic halfquadratic regularization algorithms for computed imaging, IEEE International Conference on Image Processing, Vol. 2, pp. 168-172.

[7] Delakis, I., Hammad, O. and Kitney, R.I. (2007), Wavelet-based de-noising algorithm for images acquired with parallel magnetic resonance imaging (MRI), Physics in Medicine and Biology, Vol. 52, No.13, pp. 3741.

[8] Donoho, D.L. and Johnstone, I.M. (1995), Adapting to unknown smoothness via wavelet shrinkage, Journal of the American Statistical Association, Vol.90, No.432, pp.1200-1224.

[9] Donoho, D.L., Johnstone, I.M., Kerkyacharian, G. and Picard, D. (1995), Wavelet shrinkage: Asymptopic, Journal of the Royal Statistics Society, Series B, Vol. 57, pp. 301-369, 1995.

[10] Federico, A. and Kaufmann, G.H. (2007), Denoising in digital speckle pattern interferometry using wave atoms, Opt. Lett., Vol. 32, pp.12321234.

[11] Greer, J.B. and Bertozzi, A.L. (2004), Traveling Wave Solutions of Fourth Order PDEs for Image Processing, SIAM Journal on Mathematical Analysis, Vol. 36, pp. 38-68.

[12] Grieg, G., Kubler, O., Kikinis, R. and Jolesz, F.A. (1992), Nonlinear Anisotropic Filtering of MRI Data, IEEE Transactions on Medical Imaging, Vol. 11, No. 2, pp. 221-232.

[13] Hamza, A. B., Escamilla, P. L., Aroza, J. M. and Roldan, R. (1999), Removing Noise and Preserving Details with Relaxed Median Filters, Journal of Mathematical Imaging and Vision, pp. 161-177.

[14] Hossein, R., Mansur, V., Purang, A. and Saeed, G. (2008), Speckle Noise Reduction of Medical Ultrasound Images in Complex Wavelet Domain Using Mixture Priors, EEE transactions on biomedical engineering, Vol. 55, No. 9, pp. 21522160.

[15] Kaur, A. and Singh, K. (2010), Speckle noise reduction by using wavelets, NCCI 2010 -National Conference on Computational Instrumentation CSIO, Chandigarh, INDIA, pp. 198-203.

[16] Keeling, S.L. and Stollberger, R. (2002), Nonlinear anisotropic diffusion filters for wide range edge sharpening, Inverse Problems, Vol. 18, pp 175-190.
[17] Lijima, T. (1962), Basic Theory on Normalization of Pattern", Bulletin of the Electrotechnical Laboratory, Vol. 26, pp 368-388.

[18] Ling, J. and Bovik, A.C. (2002), Smoothing LowSNR Molecular Images Via Anisotropic Median Diffusion, IEEE Transactions on Medical Imaging, Vol. 21, pp.377-384.

[19] Lysaker, M., Lundervold, A. and Tai, X.C. (2003), Noise Removal Using Fourth - Order Partial Differential Equation With Applications to Medical Magnetic Resonance Images in Space and Time, IEEE Trans. Image Processing, Vol. 12, pp. 15791590

[20] Maini, R. and Aggarwal, H. (2010), A Novel Technique for Speckle Noise Reduction on Medical Images, International Journal of Applied Engineering Research, Vol. 5, No. 1, pp. 1-8.

[21] Mansourpour, M., Rajabi, M.A.A.R. and Blais, J.A. (2006), Effects and performance of speckle noise reduction filters on active radar and SAR images, Oral Presentation at the WG I/5 and I/6 Workshop on Topographic Mapping from Space, Ankara, Turkey.

[22] Mastriani, M. and Giraldez, A.E. (2006), Kalman's Shrinkage for Wavelet-Based Despeckling of SAR Images, International Journal of Intelligent Systems and Technologies, Vol.1, No.3, pp.190-196

[23] Mateo, J.L. and Fernandez-Caballer, A. (2009), Finding out general tendencies in speckle noise reduction in ultrasound images, Expert Systems with Applications, Vol. 36, Issue 4, pp. 7786-7797.

[24] Min, L. and Xiangchu, F. (2007), Image restoration using total variation and anisotropic diffusion equation, Frontiers of Electrical and Electronic Engineering in China, Higher Education Press, copublished with Springer-Verlag GmbH, Vol. 2, No. 4, pp. 400-403.

[25] Mrazek, P., Weickert, J. and Steidl, G. (2003), Correspondence between Wavelet Shrinkage and Nonlinear Diffusion, Scale-Space 2003, LNCS 2695, pp. 101-116.

[26] Narayanan, S.K. and Wahidabanu, R.S.D. (2009), A View on Despeckling in Ultrasound Imaging, International Journal of Signal Processing, Image Processing and Pattern Recognition, Vol. 2, No.3, pp.198-203.

[27] Perona, P. and Malik, J. (1990), Scale-space and edge detection using anisotropic diffusion, IEEE Transactions on Pattern Analysis and Machine Intelligence, Vol 12, pp 629-639.

[28] Pitas, I. and Venetsanopoulos, A.N. (1990), Nonlinear Digital Filters: Principles and Applications, Kluwer Academic Publishers, 1990.

[29] Puvanathasan, P. and Bizheva, K. (2007), Speckle noise reduction algorithm for optical coherence 
tomography based on interval type II fuzzy set, Opt. Express, Vol. 15, Issue 24, pp. 15747-15758.

[30] Rajan, J. and Kaimal, M.R. (2006a), Image Denoising using Wavelet Embedded Anisotropic Diffusion (WEAD)", Proceedings of IET International Conference on Visual Information Engineering pp 589-593.

[31] Rajan, J. and Kaimal, M.R. (2006b), Speckle reduction in Images with WEAD \& WECD", Computer Vision, Graphics and Image Processing, Lecturer Notes in Computer Science (LNCS) 4338, Springer-Verlag, pp. 184-193.

[32] Rajan, J., Kannan, K. and Kaimal, M.R. (2008), An Improved Hybrid Model for Molecular Image Denoising, Journal of Mathematical Imaging and Vision, Vol. 31 No. 1, pp. 73-79.

[33] Sudha, S., Suresh, G.R. and Sukanesh, R. (2009), Comparative Study on Speckle Noise Suppression Techniques for Ultrasound Images, International Journal of Engineering and Technology Vol. 1, No. 1, pp. 1793-8236.

[34] Sun, X. and Song, G. (2007), A New Anisotropic Diffusion Equation with Adaptive Fidelity Term, International Conference on Computational Intelligence and Security (CIS 2007), pp. 330-334.

[35] Thangavel, K., Manavalan, R. and Aroquiaraj, I.L. (2009), Removal of Speckle Noise from Ultrasound Medical Image based on Special Filters: Comparative Study, ICGST-GVIP Journal, ISSN 1687-398X, Volume (9), Issue (III), pp. 25-32.

[36] Torkamani-Azar, F. and Tait, K.E. (1996), Image recovery using the anisotropic diffusion equation, IEEE Transactions on Image Processing, Vol. 5, Issue 11, pp. 1573-1578.

[37] Wang, Z. and Zhang, D. (1999), Progressive switching Median filter for the removal of impulse noise from highly corrupted images, IEEE Transaction on circuits and Systems - II, Analog and Digital Signal Processing, Vol 46, No 1, pp.78-80.

[38] Wei. G.W. (1999), Generalized Perona-Malik equation for image processing, IEEE Signal Processing Letters, Vol. 6, pp. 165-167.

[39] You, Y., Tannenbaum, W.X. and Kaveh, A.M. (1996), Behavioral analysis of anisotropic diffusion in image processing, IEEE Transactions on Image Processing, Vol. 5, Issue 11, pp. 1539-1553.

[40] You, Y.L. and Kaveh, M. (2000), Fourth-Order Partial Differential Equations for Noise Removal, IEEE Trans. Image Processing, Vol. 9, pp. 17231730.

[41] Yu, Y. and Acton, S.T. (2002), Speckle Reducing Anisotropic Diffusion, IEEE Transactions on Image Processing, Vol. 11, No. 11, pp. 1260 1270 .

\section{AUTHORS PROFILE}

Mr. K. Karthikeyan obtained M.Sc., Computer Science from Nehru Memorial College, Bharathidasan University, Tiruchirappalli, Tamil Nadu, India, in 1997, and M.Phil., Computer Science from Manonmaniam Sundaranar University, Thirunelveli, Tamil Nadu, India in 2003. He is working as Assistant Professor, in Department of Information Technology, Dr. SNS Rajalakshmi College of Arts \& Science, Coimbatore, Tamil Nadu, India. He is pursing Ph.D in Image Processing.

Dr. C. Chandrasekar completed his Ph.D in Periyar University, Salem at 2006. He worked as Head, Department of Computer Applications at K.S.R. College of Engineering, Tiruchengode, Tamil Nadu, India from 2007 to 2008. Currently he is working as Associate Professor in the Department of Computer Science at Periyar University, Salem, Tamil Nadu. His research interest includes Mobile computing, Networks, Image processing and Data mining. He is a senior member of ISTE and CSI. 